with the attendant reduction in cost (in terms of subjective distress, burden on the family, and finance) and shortened hospital stay justifies its use. Maintenance neuroleptics are employed to sustain the improvement gained. The average number of ECTs required are greater than the amount employed for depression. We also use it as an early treatment option in patients with acute psychotic episodes and in neuroleptic non-responders. The difficulty in identifying the responsive subgroup among chronic schizophrenic patients necessitates a trial for those individuals who remain symptomatic on adequate neuroleptics. The lack of long-term side-effects, the lowered dose of concomitant antipsychotics, reduction in hospital stay, and its cost-effectiveness make it an attractive treatment alternative.

A careful review of Western literature also supports many of these inferences (Taylor \& Fleminger, 1980; Brandon et al, Journal, February 1985, 146, 177-183; Dodwell \& Goldberg, Journal, May 1989, 154, 635639; Christison et al, 1991). However, their emphasis seems to be lost amidst the prevalent negative point of view. The general reduction in the use of ECT in the West appears to have resulted in an inadequate evaluation of its potential. Results from studies of few patients (fewer number of ECTs) without adequate maintenance neuroleptic medication have been 'generalised' to suggest that the treatment is not useful in the management of schizophrenia. Considering the safety of the procedure and the significant non-response to antipsychotics, a trial of ECT in schizophrenia would have to be actively contemplated. There is a definite need for a reappraisal of its potential and of attitudes among physicians towards this modality of treatment.

Christison, G. W., KRICH, D. G. \& WyatT, R. J. (1991) When symptoms persist: choosing alternative somatic treatments for schizophrenia. Schizophrenia Bulletin, 17 (2), 217-248.

TAYLOR, P. J. \& FLEMINGER, J. J. (1980) ECT for schizophrenia. Lancet, i, 1380-1382.

WEINER, R. D. (1989) Electroconvulsive therapy. In Comprehensive Textbook of Psychiatry (5th edn) (eds H. I. Kaplan \& B. J. Sadock). Baltimore: Williams \& Wilkins.

P. J. SAJU K. S. JACOB

Department of Psychiatry

Christian Medical College

Vellore 632002, India

\section{Parental age in schizophrenia in a case-controlled} study

SIR: Several studies have shown that both maternal and paternal ages at the birth of their child are increased for schizophrenics, even leading to the assertion that it is one of the few areas of schizophrenia research in which there is an impressive concordance of results (Kinnell, Journal, February $1983,142,204)$.

In previous studies (Hare \& Moran, Journal, February 1979, 134, 169-177; Kinnell, 1983) conclusions were derived after comparing the age of mother and father of patients, with those of a control population. The way the control population was constructed varies among studies but in no case was there a case-control method, and we have shown previously (Fañanás et al, 1989) that a proper stratification of the control population is a key issue in making appropriate comparisons. We report the results of a study carried out in Barcelona, where the controls were selected according to the sociodemographic variables of the patients in a case-control method.

The results refer to a sample of 120 patients and 176 controls (see Fañanás et al, 1989 for a description of the two populations and the method) and are clear cut: there is no significant difference between patients and controls in either the age of the mother (mean = 29.94 years, s.d. $=6.07$ for patients, and mean $=29.61$ years, s.d. $=6.12$ for controls; Student's $t=0.46$, $P=0.64$ ), or the age of the father (mean $=33.47$ years, s.d. $=7.48$ for patients, and mean $=33.15$ years, s.d. $=6.88$ for controls; Student's $t=0.38, P=0.71$ ).

Heterogeneity is widely accepted in the understanding of schizophrenia, and many papers have addressed the question by analysing subgroups of patients according to several variables (family history, age of onset, sex). When splitting our sample into two groups according to these criteria (presence or absence of family history of schizophrenia, early or late age of onset, female or male) no divergence was observed in the age of either parent when compared with controls.

In this study, the age of the parents is shown to be independent of the presence of schizophrenia and of the variables that may define biological subgroups.

Fañanís, L., Marti-Tusquets, J. L. \& Bertranpetit, J. (1989) Seasonality of birth in schizophrenia. An insufficient stratification of control population? Social Psychiatry and Psychiatry Epidemiology, 24, 266-270.

Laboratory of Anthropology

JAUME BERTRANPETIT LOURDES FAÑANÁS

Faculty of Biology

University of Barcelona

Diagonal 645, 08028 Barcelona

Spain 